Hargraves (Earth Planet. Sci. Lett., 10, 392 ; 1971) recently showed that some such chondrites contain a moderately strong and stable natural remanent magnetization (NRM). In this branch of geophysics, of course, the gap between principle and practice can be quite wide, as the difficulties in obtaining palaeointensities from even very young terrestrial rocks prove. Nevertheless, both Banerjee and Hargraves (Earth Planet. Sci. Lett., $17,110 ; 1972)$ and Butler (Earth Planet. Sci. Lett., 17, 120 ; 1972) have independently succeeded in determining at least minimum intensities of the primordial field and find them to be several orders of magnitude higher than commonly supposed.

Banerjee and Hargraves have used the Orgueil, Murchison and Allende meteorites which are carbonaceous chondrites representative of Wiik's (Geochim. Cosmochim. Acta, 9, 279; 1956) Types I, II and III respectively. The principal field determination was carried out using a modified form of the Thellier method (backed up by thermomagnetic, opaque mineralogy and electron probe microanalysis studies) to assess stability and identify the magnetic carriers. The Allende chondrites proved to be the most stable magnetically (thus giving the most reliable data), the magnetic carriers being very fine single-domain grains, probably of $\mathrm{NiFe}$. Even so, only temperatures up to $135^{\circ} \mathrm{C}$ could be used for the intensity determination because of thermally-induced chemical changes at higher temperature. The result of four determinations on two samples was an average palaeointensity of 1.09 oersted. The Murchison and Orgueil chondrites were relatively less stable, the usable temperature ranges being lower. Average palaeointensity values from these were 0.18 oersted and 0.67 oersted, respectively.

Butler has concentrated solely on the Allende meteorite and finds an average ancient field strength of 1.1 oersted. In a situation fraught with so many practical problems, such agreement is startling and, as Banerjee and Hargraves admit, must be regarded as fortuitous. The order of magnitude agreement is, however, important. Moreover, there are other reasons for regarding exact values with some caution. For example, the intensity determination methods used are based on the assumption that the NRM of the samples is TRM (thermoremanent magnetization). In the case of the Allende chondrite there is convincing evidence that this assumption is warranted, although in the other examples the validity is less secure. The point to bear in mind here is that TRM is the most effective magnetization process, so that if other processes were responsible for the magnetization the obtained palaeointensities would be underestimates. In other words, the values derived by Banerjee and Hargraves and Butler are at least minimum values.

Because there is no evidence to suggest that the Allende chondrite has been reheated since its formation (indeed, there is evidence to the contrary), the conclusion must be that magnetization took place in a field at least $10^{4}$ times higher than the present interplanetary field and of the order of magnitude of the present terrestrial surface field. This presumably refers to $4.44 \times 10^{9} \mathrm{yr}$ ago, the potassium-argon age of the chondrules in the Allende meteorite. As Butler points out, however, alternative interpretations are possible. The strength of about 1 oersted only applies to the ancient interplanetary field if the Allende was actually magnetized in that field. It could, of course, have acquired its magnetization in the field of its parent body. In this case, the parent planet would have required an extremely high magnetic moment at a very early stage in the formation of the Solar System.

\section{SEMICONDUCTORS}

\section{What is New?}

from a Correspondent

THE opportunity for asking this question was afforded by the celebrations in connexion with the twenty-fifth anniversary of the transistor and, in particular, in a symposium held by the Institution of Electrical Engineers in London on February 14. A small exhibition of the development of the transistor is open at IEE headquarters and is worthy of a visit.

The symposium did not forget the social aspects of the invention of the transistor, which were reviewed by Professor W. E. Farvis (University of Edinburgh); but the technical contributions and the lively discussion which followed concentrated rather on the question of the next step in the field of electronic devices. The five invited speakers were all members of research laboratories attached to leading British semiconductor manufacturers and they were all clearly adept at presenting the potential profitability of their ideas for new developments to their financial managers. They thus had an interest in presenting at least an appearance of hard-headedness. Although this rather limited the distance of the look forward and inhibited the range of an otherwise lively discussion, it allowed a firm and highly informal contemplation of the next stages in silicon technology.

The first talk was perhaps an exception to this generalization. Dr J. Evans (Standard Telecommunications Laboratories) talked about new materials and the new electronic functions which they might perform in the future. He began with an account of the versatility of gallium arsenide, which was at first used as a material for bipolar transistors but has now unexpectedly found application in light-emitting devices and as a microwave oscillator and a field-effect transistor. He held this up as an example of how to pick new materials

\title{
Soft X-ray Structure of Cassiopeia A
}

Observations of Cas A from Copernicus are reported in next Monday's Nature Physical Science (March 12) by Fabian, Zarnecki and Culhane. The diagram shows an X-ray contour map of the source in the energy range 1.4 to $4.2 \mathrm{keV}$, superimposed on the Uhuru error box for the source $2 \mathrm{U} 2321+59$. Clearly, the two objects are the same, as was already suspected.

The detail shown in the contour map is reminiscent of the first radio contour maps obtained of this source, and the overall distribution of X-rays is best explained by an annular source of outer diameter $5.5 \pm 1.5$ arc $\mathrm{min}$ and inner diameter $2.0 \pm 2.0$ arc min-this is definitely not a compact source of X-rays, even though it might have been expected that a collapsed object would have been left at the centre of this supernova remnant, which is only about 300 years old. "The data are suggestive", say Fabian et al., "of a shell source" in which X-ray generation occurs either through thermal bremsstrahlung from a blast wave or through the synchrotron process.

Much remains to be discovered from further detailed studies of the source; it is particularly interesting to compare the present X-ray observations with early radio maps of Cas $A$ and with the best detailed map now available, made at 2.7 $\mathrm{GHz}$, which the MSSL team reproduce in their article. If the accuracy of X-ray astronomy proceeds at the present pace, it may not be long before comparable high resolution X-ray maps of Cas $A$ and other extended objects are available.

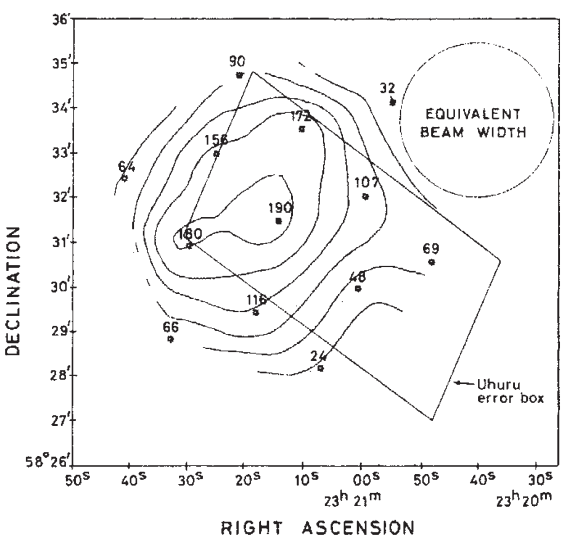

